TM-1632

\title{
Limitations to Very High Rate Wire Chambers in Fixed Target Geometries
}

\author{
Leonard Spiegel \\ Fermi National Accelerator Laboratory \\ P.O. Box 500, Batavia, Illinois 60510
}

November 1989 


\title{
Limitations to Very High Rate Wire Chambers in Fixed Target Geometries
}

\author{
Leonard Spiegel \\ Fixed Target Heavy Flavor Workshop \\ Detector Subgroup \\ Breckenridge, Co. August, 1989
}

Introduction

The task is to explore physical limitations to the operation of wire chambers in fixed target geometries at an interaction rate of $52 \mathrm{MHz}$ (chosen as it corresponds to the FNAL accelerator $\mathrm{rf}$ rate.) That is, to see if there are physical limitations to the operation of gaseous wire chambers at such a high operating rate. A primary proton beam energy of $800 \mathrm{GeV}$ has been assumed although modest increases in the primary energy should not affect the basic conclusion as secondary multiplicity increases logarithmically with centerof-momentum energy. By sustaining a $52 \mathrm{MHz}$ interaction rate it is meant that 1) results from a given interaction are electronically isolated from preceding and subsequent rf buckets (approximately $20 \mathrm{~ns}$ ), 2) good wire plane efficiency is maintained throughout the active region of the chamber, and 3 ) the chamber operates in a stable manner throughout the course of a typical fixed target run - six months to a full year.

Other working assumptions include the understanding that chamber electronics amplifiers, discriminators, delay elements, encoders - will not suffer degradation at 52 $\mathrm{MHz}$ (tacitly assumed in point 2 but not reviewed in this note) and that the intensely populated beam regions (10 tracks/rf bucket for a $10 \%$ target) have been somehow excluded from the chambers.

\section{Relationship to micro-vertex detector}

The Vertex Subgroup of the Breckenridge Fixed Target Heavy Flavors Working Group has focussed on the use of silicon microstrip detectors (SMD's) as desirable tracking devices for the region just downstream of the target or targets (decay volume for charm and beauty states.) Wire chambers, in this conceptual spectrometer design, complement SMD's by extending charged particle coverage farther downstream, where the necessary active area coverage of several square meters would argue against the costly silicon planes $\left(600 \mathrm{k} \$ / \mathrm{sq} \cdot\right.$ meter $\left.^{1}\right)$. Multiple $\cdot$ scattering and radiation length considerations 
also favor the use of wire chambers over silicon planes.

Design parameters for the SMD's determine, to some extent, the pitch or sense wire to sense wire spacing and active area of the wire chambers as one would like to preserve the angular resolution and coverage of the SMD's. Alternatively, given a minimum achievable wire pitch ( $2 \mathrm{~mm}$ ?), preservation of angular resolution dictates the minimum distance such a wire chamber may be located from the target. As an example, in the absence of a magnetic field a $2 \mathrm{~mm}$ pitch wire chamber at 12 meters has the equivalent granularity of a 50 micron SMD located 30 centimeters from target zero. Meanwhile, the $5 \mathrm{~cm}$ coverage of a typical SMD has grown to $2 \mathrm{~m}$ at the chamber. Of course other considerations, such as radiation damage to chamber wires, may dictate even more stringent positioning requirements.

\section{Cell memory time}

In order to assure that information from a particular wire cell is restricted to a single interaction it is necessary to assure that the last primary cluster electrons to drift into the sense wire do so within the $20 \mathrm{~ns}$ time allotment for the interaction. There are essentially two ways to achieve this condition: make the cells sufficiently small or by choice of gas make the electron average drift speed sufficiently high. In practice one employs a combination of both techniques to reduce the time jitter or clear-out time. For example, it is envisioned but not necessarily proven, that one can make $2 \mathrm{~mm}$ diameter straw tubes ${ }^{2}$ ( for the purposes of this discussion a straw tube is simply a long conductive tube filled with a standard gas mixture and with an anode wire suspended in the center.) Based on the maximum drift distance of $1 \mathrm{~mm}$, such a tube would require an average electron drift speed of at least $50 \mu / \mathrm{ns}$ in order to be 'cleared-out' after $20 \mathrm{~ns}$.

In addition to clearing our electrons from a passing track one must also worry about the clear-out time for the positive ions, which are drifting at a much slower average rate toward the cathode. If the density of positive ions, which depends on the gas multiplication, becomes large enough the reduction in electric field at the anode wire surface will lead to signals insufficiently large to trigger the discriminator and chamber efficiency will be lost. This loss, however, is localized to a small fraction of a wire, perhaps a centimeter, around which the positive ion cloud has developed.

Estimating loss of efficiency due to positive ion drift is not easy as it depends on the detailed mechanism of the loss and the distribution of charged tracks within the detector plane. In order to guesstimate such a loss, however, I will assume that any track which falls within $\pm 5 \mathrm{~mm}$ of a previous track along the same wire and within the next 5 buckets 
(100 ns) will be lost. This is an extremely severe assumption which nevertheless I will adopt for the purpose of illustrating that the effects of positive ion screening should be mild, especially if one is willing to retreat from the beam region.

From a PYTHIA ${ }^{3}$ simulation of $800 \mathrm{GeV}$ pp minimum bias events, one estimates that for a $2 \mathrm{~mm}$ pitch chamber $12 \mathrm{~m}$ from the target, the maximum rate should be less than 0.018 hits per linear $\mathrm{cm}$ of wire per interaction. In this simulation a horizontal momentum kick of $830 \mathrm{MeV} / \mathrm{c}$ was applied to all charged tracks at $6 \mathrm{~m}$ from target center. The distribution of intensities within the $2 \mathrm{~mm}$ horizontal by $1 \mathrm{~cm}$ vertical cells is shown in figure 1 and the projection into the horizontal axis (bend direction) is shown in figure 2 . Of course in real life one must also take into consideration the additional flux due to noninteracting beam. Either the chamber should be constructed with a 'hole' for the noninteracting beam or the central wire regions must somehow be deadened.

Combining the PYTHIA result with the above assumption leads to a local inefficiency of

$$
\eta=1-(1-0.018)^{5}=.09
$$

Clearly, though, the above assumptions are open to question and steps such as 1) increasing the drift speed of both electrons and positive ions and 2) decreasing the size of the avalanche will tend to reduce the worst case inefficiencies to tolerable levels.

\section{Fast gases}

Traditionally one has used a mixture, often 50/50, of Argon-Ethane at atmospheric pressure in large drift chambers. Argon-Ethane has the desirable property of providing, for reasonably high electric fields, a saturated drift speed. That is, above a critical electric field, the electron drift speed is only very weakly field dependent and thus, to good approximation, constant (about $50 \mu / \mathrm{ns}$ ) over the dimensions of the cell.

Within the last several years there has been considerable research ${ }^{4}$ in identifying replacements for Ar-Ethane which offer significantly higher drift speeds. These gases, which boost drift speed by reducing instantaneous electron energies, almost invariably require higher operating potentials than Ar-Ethane to achieve the same gain. However, recent advances in chamber electronics have led to an increase in amplifier sensitivity which in turn allows one to run chambers at reduced operating potentials, thus in some sense offsetting the requirement of fast gases. 
An 80/20 mixture of $\mathrm{CF}_{4} \mathrm{i}-\mathrm{C}_{4} \mathrm{H}_{10}$ (freon-14, isobutane) is an example of a fast gas alternative to Ar-Ethane. With an average drift speed of about $100 \mu / \mathrm{ns}$, this mixture is twice as fast as the standard Ar-Ethane mix, a fact which is easy to verify in the laboratory with a test cell and a time digitizer. One unfortunate by product, which should be borne in mind, is that presumably spatial resolution will be worse for the faster gas, at least for a given precision time digitizer, as a given interval of time corresponds to a factor of two greater drift distance within a cell relative to slower gases.

In addition to improved drift speed the freon-14 based gas enjoys another advantage over Ar-Ethane: the mean number of primary clusters per $\mathrm{cm}$ of gas is about twice as high in the fast gas $\left(41 / \mathrm{cm}\right.$ compared with about $\left.20 / \mathrm{cm}^{5}\right)$. A larger primary cluster density implies a smaller critical cell size necessary for good efficiency. For $\mathrm{CF}_{4} \mathrm{i}-\mathrm{C}_{4} \mathrm{H}_{10}$ a gas gap of $1.4 \mathrm{~mm}$ is sufficient to limit the inefficiency due to a lack of primary cluster to $0.2 \%$.

\section{Radiation damage}

By radiation damage in wire chambers one generally refers to the deposition of a thin dielectric film on the surface of the anode wires, the films resulting from radiation induced polymerization of organic compounds. These films lower the electric fields and thus reduce the local efficiency where they coat the wire. Similarly, dielectric films can coat the cathode elements of wire chambers such as the inner wall of the cylinder in straw tubes. In this case, however, the deposition can lead to chamber breakdown.

For standard Ar-Ethane mixtures it is known that above approximately 1 Coulomb of charge per linear centimeter of wire, effects of radiation damage lead to a pronounced decrease in pulse height from the affected region ${ }^{6}$. Recently, it has been reported ${ }^{7}$ that for the same $1 \mathrm{C} / \mathrm{cm}$ dose the $\mathrm{CF}_{4} \mathrm{i}-\mathrm{C}_{4} \mathrm{H}_{10}$ mixture suffered only $1 / 20$ the loss in pulse height.

A typical fixed target run has about $10^{7}$ seconds of beam exposure. Using the chamber model described above and the worst case estimate of $0.018 \mathrm{e} / \mathrm{cm}$, one can estimate the maximum gas gain, $\mathrm{G}$, allowed so as to limit radiation damage within a running cycle:

$$
20 \mathrm{C} / \mathrm{cm}=(0.018 \mathrm{e} / \mathrm{cm} / \mathrm{int})(6 \mathrm{pri} / \mathrm{e})(\mathrm{G})\left(510^{7} \mathrm{int} / \mathrm{s}\right)\left(10^{7} \mathrm{~s} / \mathrm{run}\right)\left(1.610^{-19} \mathrm{C} / \mathrm{e}\right)
$$

Here $G=2-310^{6}$. To be safe, one might want to run at a reduced gain, say $10^{5}$, but even this is well within the capability of chamber preamplifiers.

VI Conclusions and warning 
Although there may be many practical difficulties in making reliable $2 \mathrm{~mm}$ straw tube chamber ( $>1 \mathrm{~m}$ long wires), such tubes when coupled with a fast gas such as $\mathrm{CF}_{4} \mathrm{i}$ $\mathrm{C}_{4} \mathrm{H}_{10}$, it is not clear that there are fundamental limitations to achieving a $52 \mathrm{MHz}$ rate.

\section{Acknowledgements}

These notes grew out of discussions which took place during working sessions of the Fixed Target Heavy Flavor Workshop at Breckenridge, Co. during August of 1989. I am indebted to Dr. Walter Selove for his tireless efforts in directing the Detector subgroup and for channeling my interest in wire chambers into the problems of high rate environments.

\section{References}

[1] P. Karchin, private communication, this conference.

[2] M. Binkley, private communication, this conference.

[3] H. Bengtsson and T. Sjostrand, PYTHIA version 5.3. Software for simulating hadronic processes in high energy events.

[4] Two examples include: J. Fischer, et al., Proportional Chamber For Very High Counting Rates Based on Gas Mixtures of $\mathrm{CF}_{4}$ With Hydrocarbons, NIM A238 249-264 (1985) and D. Green, et al., Fast Non-Explosive Gases for Drift Chambers, FNAL TM-1523 (1988).

[5] J. Fischer, et al., Proportional Chamber For Very High Counting Rates Based on Gas Mixtures of $\mathrm{CF}_{4}$ With Hydrocarbons, NIM A238 249-264 (1985)

[6] Plenum session of the Workshop on Gas Calorimetry, Tuscaloosa, Alabama (1989)

[7] R. Openshaw, et al., IEEE Trans. on Nuc. Sci., NS-36, 567 (1987). 


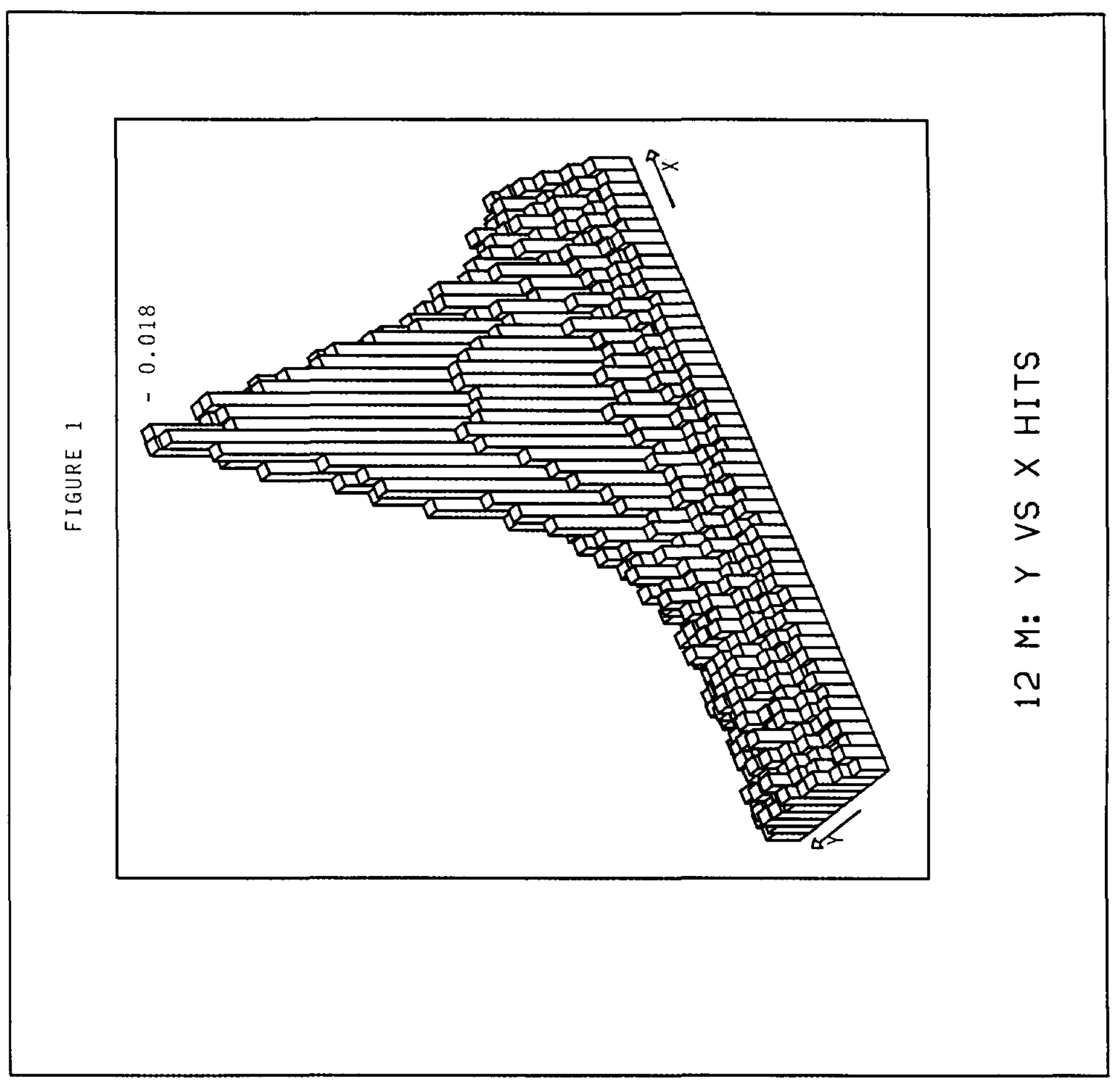




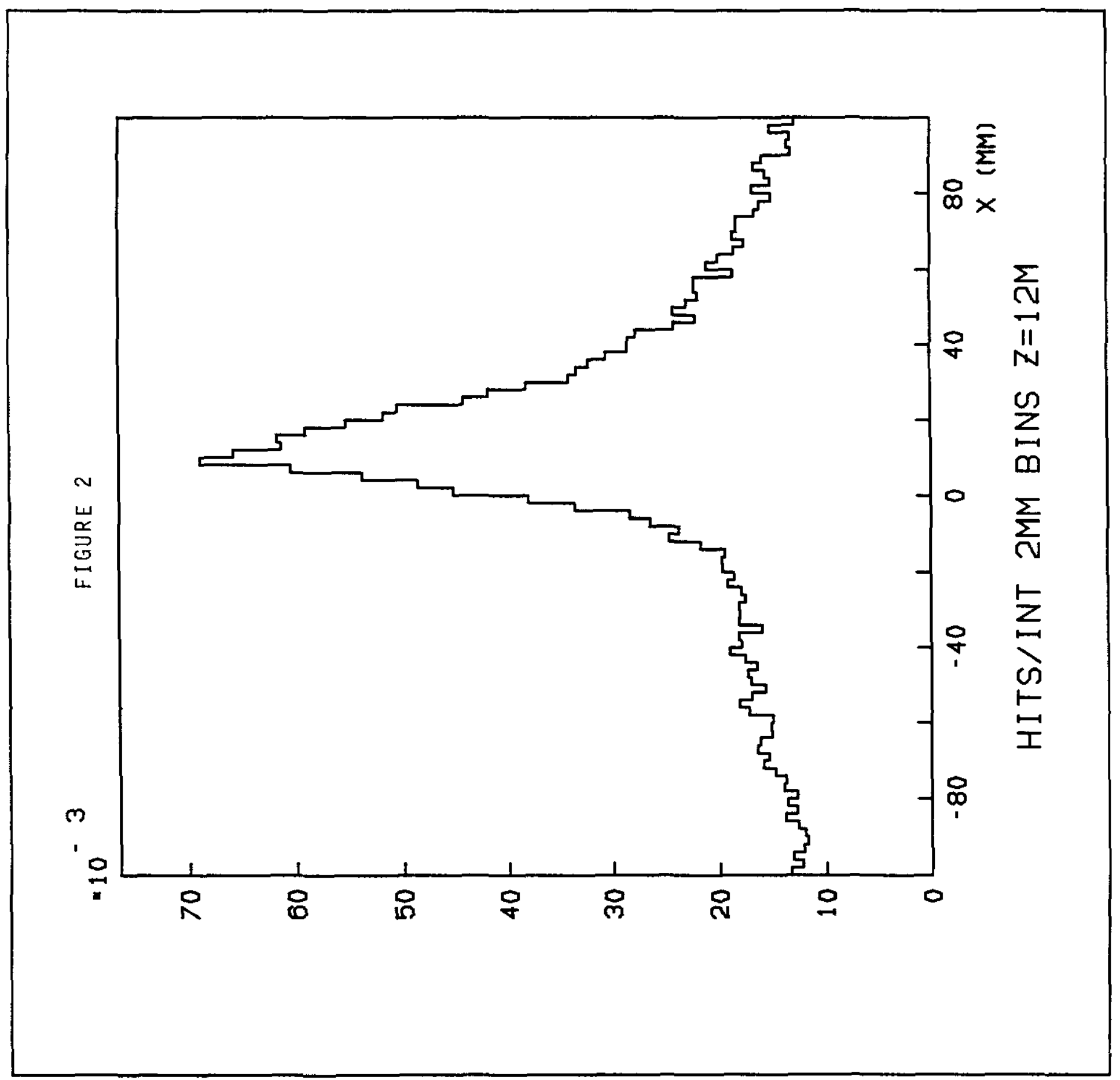

